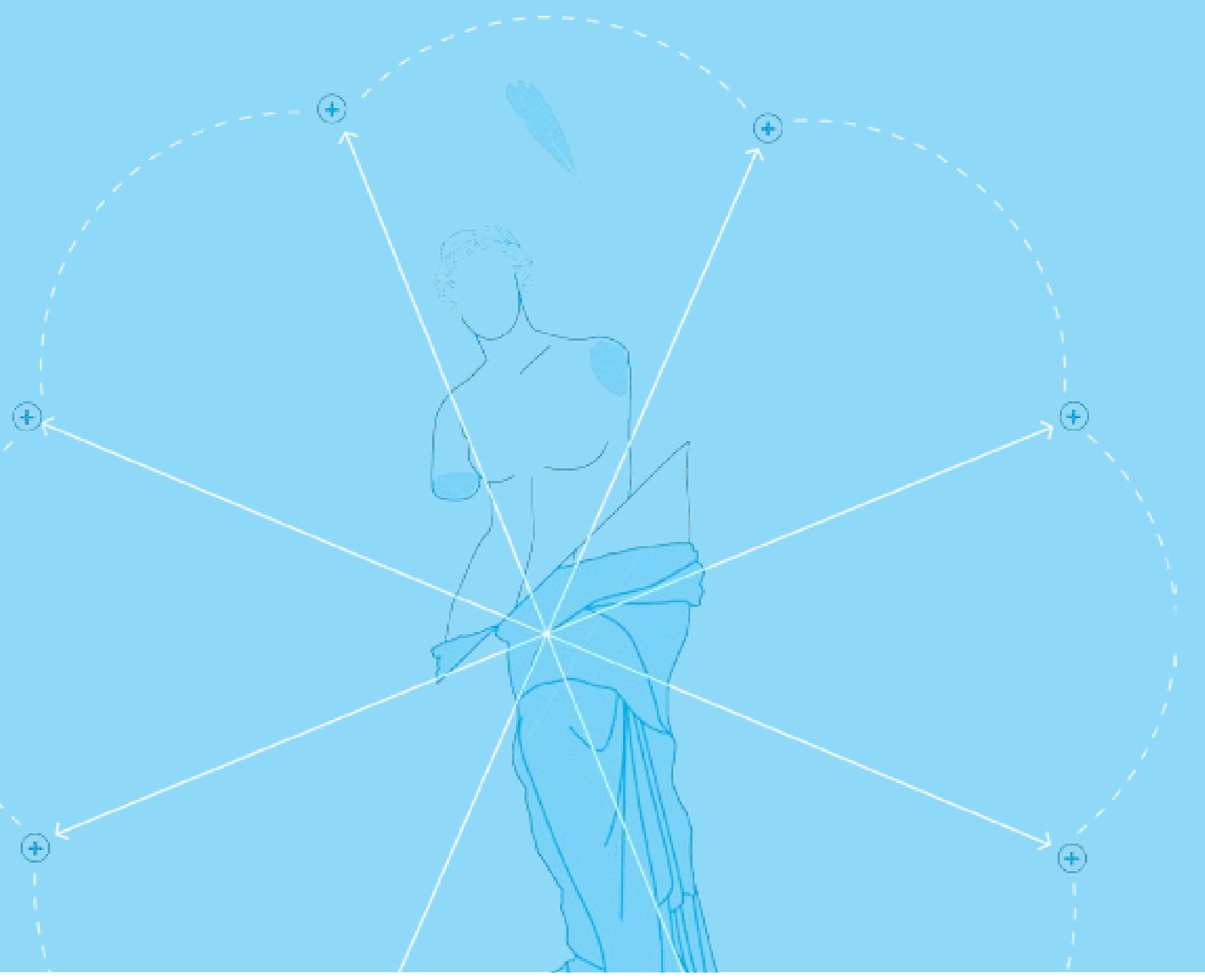

\title{
Organización de los docentes y recreación del saber
}

Organization of teachers and recreation of knowledge

Organização dos professores e recriação do conhecimento

Sigfredo Chiroque Chunga 


\section{Sigfredo Chiroque Chunga ${ }^{1}$}

1 Investigador educacional del Instituto de Pedagogía Popular(IPP), Lima, Perú. Correo electrónico: schiroque@ipp-peru.com

Fecha de recepción: 24 de febrero 2015 / Fecha de aprobación: 3 de abril de 2015.

\section{Resumen}

Profesores del Perú, organizados en pequeños grupos, han recreado la categoría "saber" y también el sentido que deberían tener las innovaciones educativas. En ambos casos, la creación de los docentes se inscribe en el marco de la Educación Popular y de la pedagogía crítica. En el presente documento se sistematiza la experiencia de esta propuesta de educación para el cambio social, la cual se ve amenazada por los avances del neoliberalismo en el Perú. Esto afecta la objetividad y subjetividad de los docentes en su proceso creativo.

\section{Palabras clave}

Saber pedagógico, innovaciones educativas, Educación Popular, pedagogía crítica.

\section{Summary}

Professors of Peru, organized in small groups, have recreated the category " know" and also the sense that they should have educational innovations. In both cases, the creation of teachers within the framework of popular education and critical pedagogy. In this document the experience of this proposal for education for social change is systematized, which is threatened by the progress of neoliberalism in Peru. This affects the objectivity and subjectivity of teachers in their creative process.

Key words:

Pedagogical knowledge , educational innovations, popular education, critical pedagogy.

\section{Resumo}

Profesores do Perú, organizados em pequenos grupos, tem recriado a categoría "saber" e também o sentido do conceito e prática das inovações educacionais. Isto se têm feito no âmbito da educação popular e da pedagogia crítica. Portanto, a experiência sistematizada neste trabalho é voltada para a mudança da sociedade e da educação que está ameaçada pelo avanço do neoliberalismo no Peru. Isso afeta a objetividade e subjetividade dos professores no seu processo criativo.

Palavras chave:

Saber pedagógico, inovações educativas, educação popular, pedagogia crítica. 
$\mathrm{C}$ on una experiencia de 30 años en el campo de la sistematización en el Instituto de Pedagogía Popular (IPP) del Perú, podemos afirmar que los docentes son capaces de recrear conocimiento y saber, no tanto como sujetos individuales, sino como colectivos organizados alrededor de "prácticas rectoras" innovadoras. Esta experiencia se plasmó en los "Círculos de Autoeducación Docente" (CADs), que han venido actuando y actúan al interior de la escuela pública. En la medida que estos grupos estaban adscritos a la Educación Popular y a la pedagogía crítica recreaban saber alternativo ("estratégico"), como parte de la creación de un nuevo tipo de sociedad y civilización.

Veamos cómo los docentes peruanos, agrupados en los CADs: a) Recrearon pedagógicamente la categoría “saber", en el marco de la Educación Popular y en coherencia con la cosmovisión andino-amazónica; y b) Crearon una manera de realizar "innovaciones educativas estratégicas", asumiendo creativamente la tesis de Leóntiev de "práctica rectora". Hagamos previamente una presentación de cómo se ha desarrollado la experiencia de esta organización docente, para posteriormente establecer los resultados de un saber pedagógico recreado desde su práctica.

\section{Círculos de autoeducación docente (CADs)}

El Instituto de Pedagogía Popular, asumió desde su creación (1984) que el Pueblo debía auto determinarse en las decisiones centrales de su vida y que esto debía hacerse un hecho también en el campo educativo. Por ello, su propuesta de "Educación Popular" fue de "autoeducación popular" con una definida teleología popular³.

2 No es casual que entre 1984 y 2011 el IPP publicase: Autoeducación. Revista de Educación Popular.

3 En la década del 80, en el Perú se perfilaron tres tendencias en la Educación Popular. Una simplemente hacía alusión a tener trabajo con los "sectores populares"; la segunda ponía el énfasis en el uso de técnicas activas y participativas; y la tercera colocaba el acento en la dimensión formativa de los sectores populares, con una finalidad histórico-política de deconstruir la sociedad capitalista y de construir un nuevo modelo de sociedad (el socialismo). La propuesta de los "Círculos de Autoeducación Docente" (CADs) asumió el tercer enfoque de la Educación Popular: la finalidad por transformar la sociedad (auto- determinada por los propios pueblos) es el marco donde se inscriben los propósitos, los contenidos, los métodos, los recursos... del proceso de autoeducación popular.
En 1986 los fundadores del IPP establecieron que una de las maneras de plasmar la autoeducación, era promover la organización de los docentes en pequeños grupos, como "Círculos de Autoeducación Docente”. Nos inspiró José Carlos Mariátegui quien, influido por las Tesis de la Internacional Socialista Roja, señaló que en cada sindicato debería existir un "círculo de autoeducación obrera" (Estatutos de la Confederación General de Trabajadores del Perú, CGTP, de 1927).

Para el año 2011, se registraron 92 CADs en seis regiones del país. Actualmente solamente llevan vida orgánica no más de 30 , pero todos aquellos que pasaron por los CADs (unos 1450 docentes) se sienten partícipes de un "Movimiento Pedagógico Popular".

\section{Momentos}

Es importante recordar los momentos por los que ha pasado la experiencia de los CADs, pues son los contextos orgánicos inmediatos que han condicionado la creación y/o recreación del saber pedagógico de estos núcleos organizados de maestras y maestros peruanos.

a. CADs, como núcleo de maestros progresistas de varias escuelas públicas que debaten lo socio-educativo. La primera experiencia de los CADs fue la de reunir a docentes progresistas que laboraban en varias escuelas o colegios. Esto se dio entre los años 1986-1990. Se debatían asuntos de la agenda educativa y/o se comentaban artículos publicados, fundamentalmente, en la revista del IPP Autoeducación y en el boletín Jornada Pedagógica, Materiales para la autoformación docente. Las reuniones tenían un carácter socio-político-educativo. No se incluían debates propiamente pedagógicos, ni mucho menos lo relacionado al quehacer cotidiano del aula. En esta etapa, los CADs sirvieron fundamentalmente para formar líderes. Muchos de los integrantes de los CADs (de esa época) devinieron en dirigentes magisteriales o intelectuales ligados al quehacer socio-educativo y político del país.

b. CADs, como núcleo de maestros progresistas de una misma institución educativa. Para fina- 
les de la década de 1980, se desarrolló una conciencia colectiva en los CADs alrededor de que se estaban formando "revolucionarios fuera del aula y reaccionarios en el aula". Se decidió buscar comportamientos liberadores dentro y fuera del aula, lo cual determinó la búsqueda orgánica de crear y/o recrear un saber pedagógico con enfoque popular y liberador, con integrantes de CADs de una misma escuela o colegio.

c. CADs, como núcleo de maestros progresistas de una misma institución educativa, pero incluyendo a los directores. La experiencia anterior excluía a los directores, pues se pensaba que eran parte "de la patronal" y representaban al “estado burgués". El debate interno de los CADs superó esta exclusión (1993-1994) y permitió un mayor nivel de influencia en la institución educativa, pero, al mismo tiempo, un ligero acomodamiento a la dinámica institucional, que no es sino la del sistema educativo hegemónico.

d. CADs con innovaciones. Aproximadamente desde 1993, se notó que los CADs se estaban convirtiendo básicamente en grupos de estudio que recreaban saber, sin considerar su propia práctica. Usaban los "materiales de autoformación docente" externos pero no creaban algo propio. A partir de este año, el boletín Jornada pedagógica de los CADs comienza a difundir una especie de nueva sección sobre "experiencias escolares" ligadas a una perspectiva popular. Ya en 1994, se postula el uso del "método de proyectos" y en 1998 se comienza a trabajar abiertamente con "innovaciones" dentro de los CADs. El planteamiento de que los CADs trabajasen prácticas innovadoras fue madurando paulatinamente, como una suerte de acción-eje que aglutinase a los miembros de los CADs. El Primer Encuentro de CADs (1995) zanjó esta posición y asumió la tesis de relacionar innovaciones educativas y revolución social. Ante la vacilación de algunos ${ }^{4}$ se acuñó el slogan: ¡Educación

4 En esta época, la represión gubernamental también llegaba a quienes hablasen de "Educación Popular", toda vez que la categoría era semejante a la usada por Sendero Luminoso, que tenía sus "escuelas populares". popular, siempre! Para no caer en las innovaciones del sistema, se hizo la diferencia entre "innovaciones funcionales" e "innovaciones estratégicas". Estas últimas se asumían como concreción del enfoque de la Educación Popular y la pedagogía histórico crítica. El saber creado y/o recreado por los CADs asumió explícitamente una connotación política.

e. De los CADs innovadores al Movimiento Pedagógico Popular. Al tomarse conciencia de que los cambios educativos y sociales no eran solamente responsabilidad de los docentes, se comenzó a trabajar de manera coordinada con otros actores sociales. En el año 2003 se creó el "Movimiento Pedagógico Popular", teniendo como núcleo central a los integrantes de los CADs, pero con la participación de padres de familia, intelectuales, Sindicato Unitario de Trabajadores en la Educación del Perú (SUTEP) y otras fuerzas. El X Encuentro Nacional de CADs (2004) dio su partida de nacimiento a esta experiencia que, como formalidad orgánica, se mantuvo hasta el 2007. Posteriormente, y hasta ahora, se mantiene latente la pertenencia a este movimiento, pero sin vínculo formal.

f. CADs: entre la cooptación y la incidencia en política educativa desde abajo. Desde inicios del 2000 la experiencia de los CADs comienza a conocerse a nivel nacional e internacional. Esto coincide con el principio de la elección formal de gobiernos en el Perú, pero también con la radicalización del modelo neoliberal y de su impacto en la educación peruana y en la conciencia de los docentes. Muchos de los integrantes de los CADs pasaron a cumplir roles de especialistas o de autoridades educativas locales o regionales, llevando consigo su experiencia de "innovadores educacionales". Esta nueva situación a veces se presenta como una "cooptación" de los integrantes de los CADs, pero, en otros casos, ha permitido tener una relativa "incidencia en políticas educativas". Este momento se profundizó entre el 2003-2007, donde se dio un mayor acento pedagógico que socio-educativo, en la dinámica de los CADs. 
g. CADs buscan recuperar el protagonismo de los niños y niñas. Entre los años 2008 al 2012 se toma conciencia de que se había ido perdiendo el protagonismo de los niños y niñas en el trabajo de los CADs. El XVI Encuentro Nacional de CADs (2011) corrige este "conductismo social" (el cambio de los maestros determina el cambio de los estudiantes), por lo que se ensayan diversas propuestas para realizar innovaciones educativas que contaran con el protagonismo de los niños y niñas.

h. Los CADs se asumen como "movimiento" de resistencia en el contexto de la hegemonía neoliberal. En los últimos 10 años, diversas condiciones impuestas por el neoliberalismo han generado que los docentes en general no tengan tiempo para reuniones y tomen distancia de las organizaciones formales. Esta situación ha hecho que también los integrantes de los CADs se dispersen; sin embargo, un 90\% de ellos mantiene su opción por la Educación Popular, al punto de que el XVIII Encuentro de CADs (2013) se realizó de manera autofinanciada. Allí se decidió mantenerse como un movimiento conformado por docentes "sueltos" y CADs, teniendo como condición la determinación por la búsqueda de un nuevo tipo de sociedad y civilización. Los participantes del evento decidieron centrar su debate interno en dos aspectos fundamentales: 1) Educación Popular y socialismo "sin calco ni copia” y 2) Vigencia de la Educación Popular en el contexto neoliberal; rol del docente. Este debate llega hasta la actualidad (2015), buscando alternativas en un nuevo contexto de hegemonía neoliberal (privatización de la educación en marcha, nueva subjetividad de los actores sociales, debilitamiento de la organización sindical, etc.).

\section{Enfoque de saberes}

Una de las opciones que hemos tomado en conjunto con los CADs ha sido la de recrear la categoría saber. Asumimos esta categoría haciéndola coincidir con la cosmovisión andino-amazónica así como con el sentido griego de sophía que va más allá de la gnose (conocimiento). Resumimos la connotación de "saber" que estamos usando de la siguiente manera.

Inicialmente, recuperamos los planteamientos de Juan Pascual Leone, quien habla de "saber objetivo, subjetivo y objetivado". Posteriormente, corregimos y ampliamos los postulados de Leone y, en lugar de hablar de un "saber subjetivo", postulamos un "saber subjetivado o aprendizaje", agregando, además, un "saber recreado".

Aceptamos que históricamente las diversas experiencias humanas se fueron organizando internamente en las personas como "representaciones mentales o pensamientos y conocimientos; como afectos-sentimientos-valores"; también como formas de ejercicio de "autonomía y libertad". Fue así que surgieron, como parte del desarrollo de la personalidad de cada sujeto y como una histórica producción social: los conocimientos, los sentimientos-valores y las formas de actuar con libertad. Desde allí, y teniendo soportes naturales en cada persona, se conforman: estructuras cognitivas, estructuras afectivo-valorativas y estructuras conativas.

Las creaciones humanas, entonces, no solamente son objetos materiales, tangibles y visibles; sino realidades no-materiales. Todo ello constituye la cultura, la cual conlleva una objetividad y una subjetividad. La producción cultural, entonces, globaliza una objetividad cultural y una subjetividad cultural.

\section{Saber objetivo}

El conjunto de conocimientos, sentimientos y formas de actuar en los humanos viene a constituir una producción cultural generada dentro de los sujetos individuales y colectivos. La tradición oral, los textos, grafos y otras formas surgieron como depositarias de esta producción de los humanos. Ortiz Cabanillas denominó a esta creación humana no-material, como "información social" (socialmente creada) dentro del sujeto. Ella se diferencia de la información "genética" (interna a los seres vivientes, pero connatural a ellos) y de las creaciones humanas materiales.

El desarrollo de las creaciones humanas dentro de sí (en lo afectivo, en lo cognitivo y en las formas de ejercicio de la libertad) constituye la construcción de la actividad consciente humana y, por lo tanto, es la base del desarrollo de su personalidad y, aún más, de su propia tipicidad como humano. Qué importante es reconocer que la actividad educativa hace posible sistemática- 
mente esta formación de la conciencia humana y de su personalidad, como lo explica el neurocientífico peruano Ortiz Cabanillas.

Aunque algunos le llaman "cultura", convencionalmente hemos denominado Saber, social e históricamente producido-acumulado en cada pueblo, al conjunto articulado de conocimientos, de sentimientos-valores y de formas de actuar en libertad; preferimos usar la categoría "saber" por dos razones: a) La cultura comprende la objetividad y subjetividad de las creaciones humanas y estamos asumiendo que el saber corresponde básicamente a la subjetividad; y b) El saber o sabiduría (sophía, en griego) es una categoría más amplia que el "conocimiento" (gnose, en griego).

Durante su propio desarrollo, la Humanidad fue organizando y clasificando su saber en disciplinas, dimensiones, áreas, tópicos, etc. Además sobre él fue guardando "información social" fuera de sí, lo cual constituyó el saber objetivo como derivación de lo que Vigotzky llama procesos psíquicos "interpersonales".

\section{Saber subjetivado: Aprendizaje}

Sin embargo, hay que señalar que somos humanos en cuanto pertenecemos a una colectividad. De allí que los procesos externos, de interacción de los sujetos o interpersonales, generan dialécticamente procesos psíquicos intrapersonales: El saber objetivo se internaliza en las personas y se transforma en saber subjetivado o aprendizaje. Este tránsito se da por experiencia directa, no mediada intencionalmente, o por experiencia mediada intencionalmente.

El aprendizaje intencional históricamente se generó cuando se dio la separación entre la producción cultural y la reproducción-recreación cultural. Cuando esto sucedió, el tránsito del saber objetivo hacia el saber subjetivado tuvo el prerrequisito de la mediación. Para decidir "qué" del saber objetivo se convierte en un saber subjetivado -considerando un "para qué"- hay sujetos decisores que definen lo que se debe enseñar y aprender; estos sujetos decisores actúan con historicidad, según el contexto de poder hegemónico.

El saber objetivo, organizado como información social, se comportó inicialmente como un "valor de uso" de las personas. Lo que se enseñaba y aprendía, respondía y se orientaba fundamentalmente a las necesidades de las prácticas humanas. Sin embargo, cuando surgen modelos de sociedad clasista, el valor de uso del saber objetivo se va transformando en valor de cambio o mercancía (Paro, 2006).

EducAción y CIUdAd No 29 Julio - Diciembre de 2015 ISSN 0123-0425 - PP 50 - 60
Las decisiones sobre los contenidos curriculares no se hacen espontáneamente. La selección de los mismos, antes que ser una tarea técnico-pedagógica, es fundamentalmente socio-política, pues esos contenidos deben guardar coherencia con el "para qué" enseñar o aprender. Sirven para establecer qué del saber objetivo debe servir para la reproducción y recreación cultural establecida por los grupos de poder.

\section{Saber Objetivado}

¿Pero de qué valen los aprendizajes, si ellos no se aplican, ni se concretizan?

Desde diversos enfoques, se ha insistido en una "educación para la vida". El mismo uso de un "currículo por competencias" indirectamente señala que los aprendizajes deben traducirse en un "saber hacer". Para los CADs, todo aprendizaje debería culminar estableciendo dónde y cuándo poder aplicarlo, pero dentro de un explícito "para qué". Importa, entonces, avanzar hacia el saber objetivado, donde postulamos que un articulado saber subjetivado (como "aprendizajes sintéticos"5) se aplique y sirva para la transformación de la vida en la práctica social.

$\mathrm{Al}$ aplicarse el saber objetivo, transformado en saber subjetivado, en la práctica social se permite que los aprendizajes adquiridos e interiorizados por los sujetos individuales y colectivos tengan un valor de uso. Esto, en la medida en que la práctica educativa se ha ido convirtiendo en mercancías: no solamente los servicios educativos, sino el contenido de los mismos se han ido consolidando en su valor de cambio. Es decir, el saber objetivado ha devenido en simple mercancía, aunque podría ser usado también como instrumento de liberación. Desde los sectores populares, importa -con plena conciencia histórica- recuperar el saber objetivo acumulado y el saber subjetivo para aplicarlo y usarlo en función a sus necesidades e intereses inmediatos y estratégicos. Como señala Vítor Paro:

Si esa conciencia histórica se junta con la percepción de la importancia que representa para la clase trabajadora la aprehensión del saber como instrumento de transformación social, en-

\footnotetext{
5 En el enfoque curricular vigente en el Perú, no solamente el proceso lleva a simples "logros de aprendizaje" ( que los aprendizajes son meramente acumulativos y yuxtapuestos. Los "aprendizajes sintéticos" suponen que las diversas disciplinas o áreas curriculares dialogan entre sí y forman un corpus articulado de saberes para resolver situaciones concretas.
} 
tonces, se explicitaría una verdadera dimensión revolucionaria del trabajo pedagógico en la escuela" (Vítor Paro, 2002, p. 118).

En realidad, el uso del saber objetivo como contenido en el enseñar y aprender, así como su aplicación en la práctica social, tiene que ver con la finalidad educativa. La finalidad como que se cumple al término de un proceso; sin embargo, ella se fija al inicio del proceso y tiñe todo el proceso (finis est primus in intentione, ultimus in executione). Los grupos de poder guardan celosamente su autonomía para señalar los fines educativos y curriculares. Gimeno Sacristán dirá:

El currículo es un texto que representa y presenta aspiraciones, ideales y formas de entender su misión en un contexto histórico muy concreto, desde donde se toman decisiones y se eligen caminos que están afectados por las políticas generales, las económicas, la pertenencia a diferentes medios culturales, etc. Lo cual evidencia la no neutralidad del contexto para el texto y el origen de desigualdades entre individuos y grupos (Gimeno Sacristán, 2010, p.15).

Procesos de enseñanza y de aprendizaje que se quedan en la intrasubjetividad (logros de aprendizaje) ni siquiera tienen sentido para los teóricos del sistema hegemónico. En términos estratégicos, la finalidad educativa se plasma en el saber objetivado, pero, al mismo tiempo, ella pre-condicionó la selección y organización del saber objetivo y del mismo saber subjetivado. Cuando no se explicita esta finalidad social, se está asumiendo que simplemente corresponde a la simple reproducción y/o mejoramiento del modelo de sociedad que tiene hegemonía.

Cuando los integrantes de los CADs aceptan que los aprendizajes deben concretizarse, se genera un importante tránsito: descubren que la aplicación de los saberes sirve para transformar las situaciones dadas-existentes en la realidad. Todo aprendizaje debe servir para cambiar algo de uno mismo, del conjunto social o de la naturaleza. Los docentes de los CADs llegan a la conclusión de que -en la misma ontogénesis del quehacer educativo (por su finalidad)- se encuentra una propuesta de transformación y de liberación: Aplicar el saber subjetivado para transformar "situaciones-que-son" en "situaciones-que-deben-ser". De manera interesante, llegan a una opción por la pedagogía crítica, la educación liberadora y comprometida con la transformación.

\section{Saber Recreado}

En la práctica de los CADs, se asume que todo aprendizaje se da en sujetos. Aprenden sujetos estudiantes, sujetos docentes y sujetos sociedad. Por ello, en la medida que se asumen como sujetos individuales y/o colectivos, ellos reproducen el saber aprendido a su manera, con un "plus" que deviene de su propia experiencia acumulada y de sus potencialidades particulares. Necesariamente recrean el saber objetivo y subjetivado,

Por otro lado, las necesidades humanas no son estáticas y su evolución tiene fundamento natural y cultural; por ello, las prácticas humanas y sus experiencias resultan cambiantes. Nuevas necesidades, reales o impuestas, de los humanos, han ido surgiendo en todos los campos y han generado la necesidad de tener recreación cultural.

En este marco, el proceso educativo no se agota en la simple reproducción, repetición y aplicación del saber existente. Desde la Educación Inicial, debe promoverse el hábito creativo, no solamente en creación material, sino también en el campo de los conocimientos, en la manifestación de los sentimientos-valores y en las formas de ejercicio de la libertad. Cada vez es más claro que el saber subjetivado y objetivado se complementa con el saber recreado que finalmente realimenta al saber objetivo. Ver Gráfico 1.

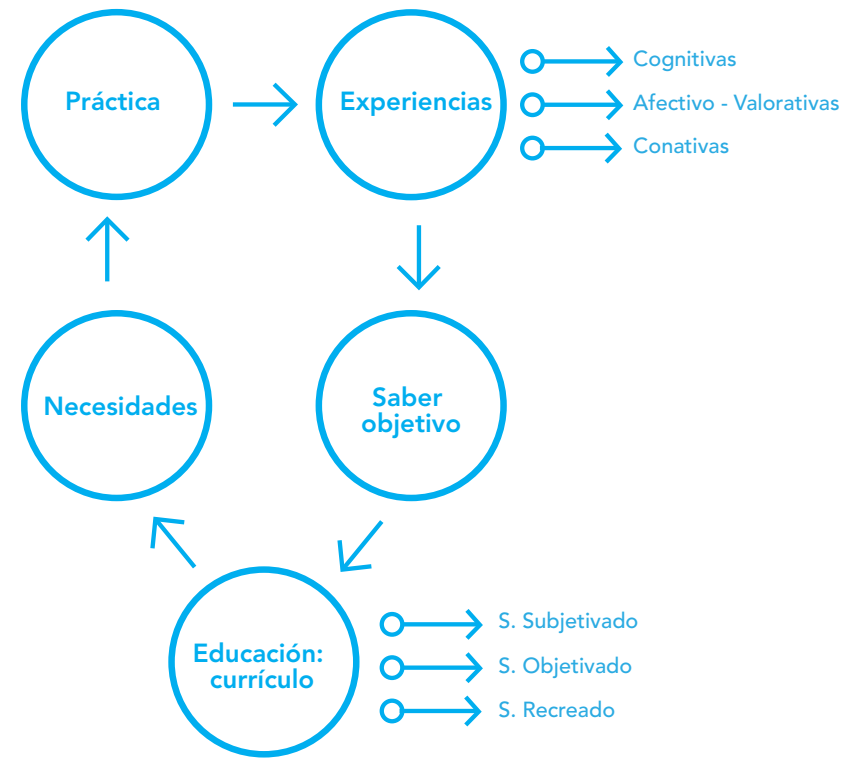

Gráfico 1.

Organización de los docentes y ReCreación del saber - Sigfredo ChiRoque Chunga 


\section{Práctica del enfoque}

El recreado enfoque de Saberes que hemos resumido -al concretizarse en el quehacer educativo- viene generando múltiples consecuencias:

- Atención holística de la formación. Los aprendizajes ya no giran meramente en lo cognitivo; sino que deben verse también en lo afectivo-valorativo y en lo conativo o formación para el ejercicio de la libertad (toma de decisiones).

- Opción crítica. La orientación de buscar superar "situaciones-que-son" para llegar a "situaciones-que-deben-ser" no solamente devino de una opción inicial por la Educación Popular y la pedagogía crítica. Se consolidó cuando se postuló que todo saber debe ser "objetivado" y "recreado". De manera interesante, los docentes recrearon el "método de la problematización" como una herramienta que ha permitido promover un hábito de cambio en ellos mismos y en sus estudiantes.

- Conciencia crítica y no solamente crítico. Inicialmente, en los CADs, se asumió como un avance el "pensamiento crítico". Posteriormente, se fueron explicando las limitaciones del uso de esta categoría, por su acento meramente cognitivo. Un paso intermedio fue hablar del "desarrollo de la criticidad" (cognitiva, afectivo-valorativa y en la toma de decisiones), y, con el apoyo de la neurociencia, hablar del crecimiento de la "personalidad", en la medida que se promueve el desarrollo de sus estructuras afectivo-valorativas, cognitivas y conativas; esto es, el desarrollo de la "conciencia" personal. En este marco, al interior de los CADs ya no se habla de la necesidad de un "pensamiento crítico", sino de una "conciencia crítica".

- Reencuentro con la cosmovisión andino-amazónica. En la cosmovisión andino-amazónica, toda práctica humana debe orientarse para arribar al Allin Kawsay (Buen Vivir). Pero, para llegar a esta meta deseable, necesariamente uno tiene que vivenciar el: Allin Munay
(Querer bien), Allin Yachay (Pensar bien) y Allin Ruray (Tomar decisiones y trabajar bien). En esta cosmovisión el punto de partida es el Allin Munay. $\mathrm{Al}$ reconocer y comparar esta cosmovisión, con el enfoque de Saberes, se encontraron similitudes con algunas variantes que han permitido replantear el trabajo educativo-popular. Se estableció que -en las sesiones de aprendizaje- era preferible partir de un "querer" aprender y no tanto de un "conflicto cognitivo". Así, se asumió la tesis de armonía "consigo mismo", "con los demás" y con la "naturaleza" que es parte de la cosmovisión andino-amazónica. Aún más, se han comenzado a revisar las bases epistemológicas de la misma Educación Popular. En las décadas del 70, 80 y 90 se aceptaba acríticamente que el humanismo marxista y el humanismo cristiano constituían las bases de la Educación Popular. Ahora, en los CADs, se habla de otro fundamento necesario: el humanismo originario. No hacerlo sería aceptar una suerte de neocolonialidad progresista del saber.

- Proyecto Curricular Regional de Puno. Desde el 2007 en el Perú se acepta formal y legalmente que debe existir un Marco Curricular Nacional (MCN) y unos Proyectos Curriculares Regionales. En la práctica, el MCN todavía está en debate, y no existen PCRs. Solamente la Región de Puno elaboró su PCR, con el criterio de "construcción social" y como un "Currículo por Saberes" (20062011). Su propuesta se viene ejecutando, aunque con el enojo de las autoridades de Lima. De manera significativa, la recreación del enfoque de $\mathrm{Sa}$ beres iniciada de los CADs ya está alimentando decisiones pedagógicas de alcance regional.

- Cultura investigativa en Educación Básica. Asumiendo el enfoque Saberes, en los últimos años se viene promoviendo el desarrollo de una "cultura investigativa” desde la Educación Básica. Una forma sistemática de recrear el saber es la de ir formando a los estudiantes y docentes para una práctica investigativa desde la Educación Inicial o parvularia. Ya se cuentan con "carteles" que experimentalmente se vienen validando, así como 
con una propuesta inicial de promover investigación desde la Educación Básica, con enfoque intercultural.

\section{Innovaciones educativas}

Los CADs han surgido con una clara y sistemática opción por el cambio social y educativo. Por ello, desde su creación buscaron concretizar esta opción. Inicialmente, se constituyeron como "grupos de estudio", pero esto implicó algunas limitaciones: La de ser revolucionarios en la calle y reaccionarios en el aula; la de ser repetitivos de los contenidos curriculares del sistema en su práctica docente sin tener alternativas prácticas como educador popular en el aula; y la de tener una pertenencia pasajera en los CADs, en la medida que el simple compromiso intelectual no daba suficientes nexos a la vida orgánico-pedagógica.

Como en otros países de América Latina, en la década del 90 también en el Perú los docentes fueron invitados a ser "innovadores". Los integrantes de los CADs analizaron la iniciativa que venía del Ministerio de Educación y establecieron que ésta coincidía con las prácticas innovadoras que venían realizando hacía muchos años, pero con una diferencia: se pedía hacer innovaciones para mejorar los procesos y resultados del sistema.

En los CADs se había establecido que existía una innovación educativa cuando se alteraban los procesos y/o los insumos para resolver algún problema, sea de enseñanza, sea de aprendizaje o cuando se usaban renovados procesos y/o insumos para conseguir algún objetivo, meta o resultado educacional.

Los integrantes de los CADs tenían claro que -desde una opción por la Educación Popular y la pedagogía crítica- debían hacer innovaciones para modificar el sistema educativo y social. El Ministerio les pedía hacer innovaciones para mejorar el sistema. Tomaron dos decisiones importantes:

- Diferenciar las "innovaciones funcionales" (al sistema) de las "innovaciones estratégicas" (que buscaban y buscan alterar el sistema).

- No asumir como antagónicos los dos tipos de innovaciones antes señaladas, sino pensar que las "estratégicas" "iban más allá de las funcionales". De esta manera, fue posible trabajar sin mayores cuestionamientos de las autoridades oficiales.

\section{Priorización de capacidades y procesos desencadenantes}

En realidad, en las innovaciones educativas de los CADs se busca que se plasmen algunas capacidades priorizadas para ser trabajadas con los estudiantes. La priorización se hizo asumiendo tres criterios:

- Que apuntasen a la transformación social y educativa.

- Que recogiesen el "saber" de nuestros pueblos, no como una táctica para acabar imponiendo el saber occidental; sino valorando este saber.

- Que en todos los casos se trabajase lo afectivo-valorativo, lo cognitivo y la formación para el buen ejercicio de la libertad.

En el Cuadro 1, se precisan las capacidades que han sido priorizadas en los últimos años (2012-2015).

\section{CAPACIDADES PRIORIZADAS EN EL TRABAJO CON LOS NIÑOS/AS. SE CONVIERTEN EN "ACTIVIDAD RECTORA"}
$1 \rightarrow$ Formación en derechos del niño y la niña: equidad de género.
$2 \rightarrow$ Desarrollo de la criticidad y la creatividad.
$3 \rightarrow$ Desarrollo de la cultura investigativa.
$4 \rightarrow$ Promoción de hábitos organizativos y liderazgo.
$5 \rightarrow$ Desarrollo de adecuados hábitos alimentarios.
$6 \rightarrow$ Creación de entornos lúdicos.
$7 \rightarrow$ Desarrollo de capacidades para el trabajo productivo.
$8 \rightarrow$ Relación amigable con la naturaleza (Pachamama).
$9 \rightarrow$ Interculturalidad.

Cuadro 1. 
En la experiencia que se tiene con "Círculos de Autoeducación Docente", partimos de que el comienzo de toda acción o práctica es el acto afectivo-volitivo de "querer y tomar decisiones", recién entonces deviene el "pensar". Cuando uno llega a un grupo de docentes, mueve su sentimiento para que decidan hacer algo nuevo en su institución educativa; es decir, promueve la ejecución de alguna "innovación educativa" (Ver Gráfico 2).

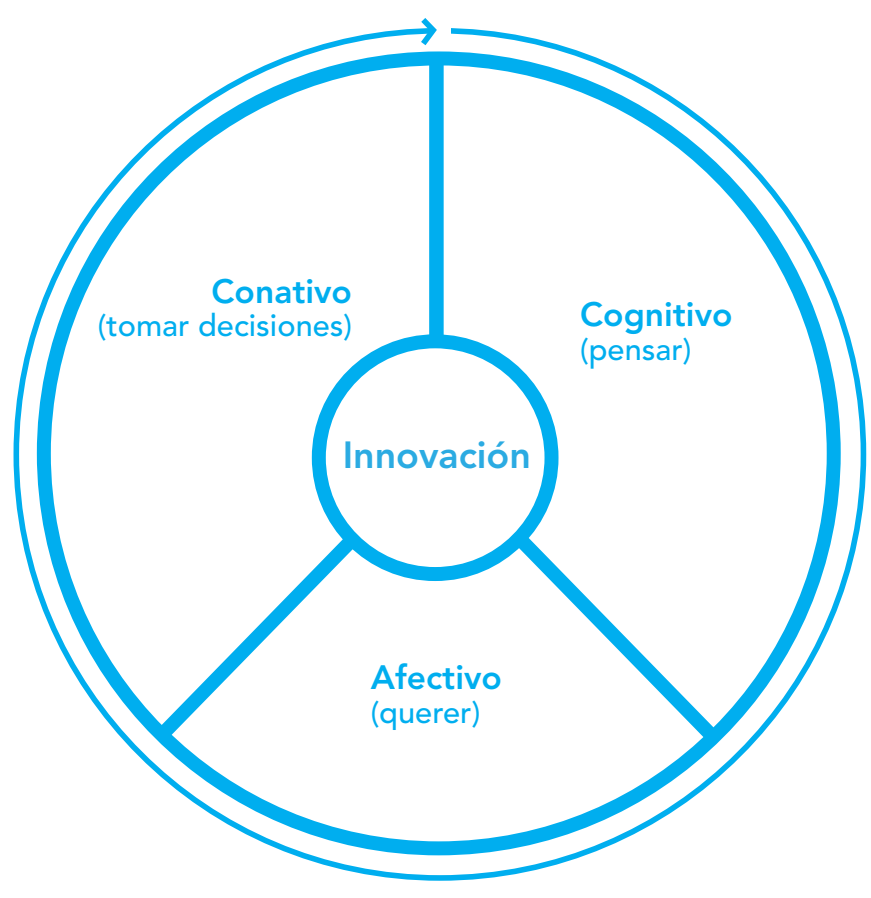

Gráfico 2. Procesos desencadenantes de la práctica innovadora

"Después" de que uno quiere y toma la decisión de hacer algo, recién se colocan los prerrequisitos cognitivos y los prerrequisitos organizativos. Obviamente este no es un proceso mecánicamente secuencial, pero en la experiencia de los CADs se ha venido utilizando exitosamente.

Solamente después de este "querer" y "tomar decisiones", se discute cuáles son los prerrequisitos formativos y los prerrequisitos organizativos para concretizar la "innovación educativa". La misma estrategia se sigue con los niños y niñas. Con ellos se discute "la innovación educativa" que se va a poner en marcha y, obviamente, los pasos preliminares son: "querer y tomar decisiones".

La práctica innovadora no se escoge al azar, sino que guarda relación con alguna de las capacidades estratégicas seleccionadas. Resulta siendo el "campo" o "situación concreta" donde los niños y niñas desarrollan alguna capacidad central. En la innovación, el niño debe asumir el protagonismo; por ejemplo, en una experiencia de "liderazgo", el proceso formativo del niño o niña líder se da en la medida en que ellos van "salón por salón" enseñando a sus coleguitas acerca de los Derechos del Niño y Niña o sobre la Equidad de Género.

En cada CAD se analiza el contexto y se determina qué capacidad se va a priorizar para, a partir de esto, escoger la práctica innovadora (innovación educativa) con la cual se va a trabajar. En la medida que se está trabajando con "capacidades estratégicas", las innovaciones adquieren la misma connotación y apuntan al cambio: al Desarrollo Humano, en la perspectiva del Buen Vivir, deconstruyendo saberes que condicionan la manutención del sistema y promoviendo saberes que coadyuvan al cambio. Por ejemplo, en los CADs de área rural norandina se viene promoviendo la formación de "ronderitos escolares", en un contexto donde los adultos están organizados en "rondas campesinas", como formas de autodefensa.

\section{Innovación, como práctica rectora}

En la experiencia de los CADs, las innovaciones aparecieron como "prácticas" innovadoras o situaciones concretas que permitían el desarrollo del saber subjetivado, objetivado y recreado. Allí era posible la articulación de los saberes de diversas áreas curriculares, permitiendo un "aprendizaje sintético" y aplicado".

Los CADs vienen trabajando con innovaciones educativas desde hace más de 10 años. En este tiempo, nos dimos cuenta de que trabajábamos de manera yuxtapuesta y acumulativa en las capacidades. Un año se trabajaba el tema de Derechos Humanos, el año siguiente el de alimentación, después el de liderazgo y así sucesivamente. ¡Como si la cuestión alimentaria o de Derechos Humanos se diese solamente en un año determinado!

El estudio de las tesis de Leóntiev sobre la "práctica rectora" -que prevalece en determinados tramos de edad, articulando las demás prácticas- nos dio pistas para superar el problema que teníamos. Desde la experiencia de los niños y niñas, con el acom- 
pañamiento de los integrantes de los CADs, se ha ido generando una interesante propuesta que hemos denominado "trabajo por ejes", uno de los cuales se convierte en "actividad rectora". Es decir, una innovación central sirve de eje para articular el desarrollo de otras acciones relacionadas a otras capacidades.

Una innovación educativa central es asumida como actividad rectora en la medida que articula, motiva y desencadena otras prácticas. Leóntiev nos habla de las "prácticas rectoras" asociadas a las acciones centrales de un tramo de edad. En nuestro caso, hemos recogido el legado de Léontiev y Vigotzqy sobre la práctica, pero lo hemos aplicado al caso específico de las prácticas innovadoras que ejecutan los niños y niñas, con el acompañamiento de los integrantes de los CADs.

En la práctica concreta, se trata de seleccionar una capacidad estratégica para trabajarla con los niños y niñas. Como hemos dicho, esta capacidad se convierte en innovación, la cual es asumida como "actividad rectora" y colocada en el centro. Ella permitirá que se realicen diversas actividades de otras capacidades, pero girando alrededor de la innovación priorizada.

Desde el diálogo con los niños y niñas cada CAD prioriza la innovación que va a poner en el centro y cómo va a hacer para que haya otras actividades (correspondientes a otras capacidades) que "bailen", alrededor de la principal; como en los bailes de cumpleaños: todos bailan, pero alrededor de la persona que cumple años, que es diferente según las circunstancias.
En conclusión, podemos aseverar que -desde la práctica- núcleos organizados de maestros y maestras vienen produciendo y/o reproduciendo saber pedagógico, en un marco de la Educación Popular y de la pedagogía crítica dentro del aula. Sin embargo, como hemos dicho, la imposición neoliberal está generando significativos cambios en las condiciones de trabajo de los docentes, así como una nueva subjetividad en ellos. La producción y reproducción de saber pedagógico, de los docentes que han optado por la Educación Popular, se viene resintiendo.

No solamente se está presentando un problema de "contextualizar" la experiencia; sino de precisar el horizonte de sociedad y de educación que sirve como referencia estratégica para seguir produciendo y/o reproduciendo saber con perspectiva histórica. La aseveración de una "educación para la transformación social" resulta limitada. La crisis del "socialismo realmente existente" genera nuevos retos para la creación y/o recreación del saber pedagógico de los docentes comprometidos. Más allá de aportar en renovados procesos y recursos educativos, se trata de comenzar a aportar en cuanto a perfilar mejor la utopía de nuestros pueblos.

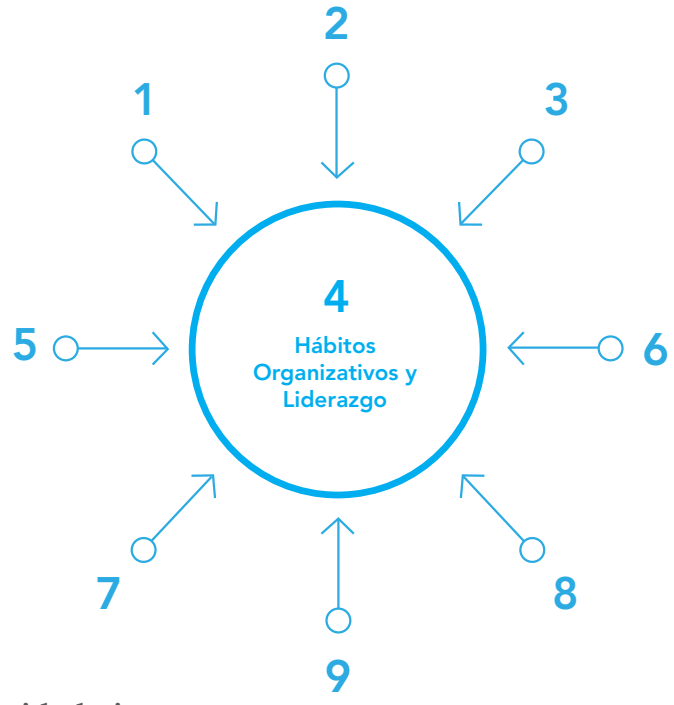

Gráfico 3. Innovación, desde una capacidad-eje 


\section{Referencias}

Ansión, J., et al. (2013). Investigación desde el trabajo docente. Lima: Instituto de Pedagogía Popular.

Antunes, Á., et al. (2010). Pedagogía crítica: Metodología y estrategia. Lima: Instituto de Pedagogía Popular.

Baquero, R. (1997). Vigotsky y el aprendizaje escolar. Buenos Aires: Aique.

Bourdieu, P. (1998). Escritos de educaçao. Petrópolis: Vozes.

Caro, R. G. (1975). Las Escuelas de estudio y trabajo en educación. Lima: Asencios.

Carr, W., y Kemmis, S. (1998). Teoría de la crítica de la enseñanza. Barcelona: Martínez Roca.

Carretero, M. (1993). Constructivismo y educación. Buenos Aires: Aique.

Chiroque, S. (2007). Cuando las innovaciones educativas se validan y sistematizan para convertirse en propuestas. Lima: Instituto de Pedagogía Popular.

Chiroque, S., y Céspedes, G. (2012). Los niños y las innovaciones educativas para el cambio, desde la experiencia de los CADs. Lima: Instituto de Pedagogía Popular.

Chiroque, S. (2013). Sindicalismo docente en el Perú: ¿Refundación en el marco neoliberal? En Reflexión e Investigación. Revista Editorial del Congreso por una Educación de calidad, No. 5. Barranquilla: Fondo Editorial del Caribe.

Coll, C., et al. (1997). El constructivismo en el aula . Barcelona: Grao.

Gimeno Sacristán, J. (Comp.). (2008). Educar por competencias: ¿Qué hay de nuevo? Madrid: Morata.

Huanacuni, F. (2010). Vivir bien/Buen Vivir. La Paz: Convenio Andrés Bello e Instituto Internacional de Integración.

Leone, J. P. (1997). Constructivimo dialéctico como fundamento epistemológico de la ciencia humana. En Ospina, H., y López, L. Estrategias constructivistas, pedagogías activas y desarrollo hu-

Educación y CIudad No 29 Julio - Diciembre de 2015 ISSN 0123-0425 - PP 50 - 60 mano. Bogotá: I Encuentro Internacional y IV Encuentro Nacional de Pedagogía activa y Desarrollo Humano.

Leóntiev, A. N. (1975). Actividad, consciencia, personalidad. Moscú: Universidad Estatal de Moscú.

Leóntiev, A. N. (1983). Obras escogidas. Moscú: Pedagogía.

López de Castilla, M., y Chiroque, S. (2004). Innovaciones educativas para el cambio. Lima: Instituto de Pedagogía Popular.

Ortíz, P. (2004). El nivel consciente de la Actividad Personal. Lima: Universidad Nacional Mayor de San Marcos.

Ortíz, P. (2008). Educación y formación de la personalidad. Lima: Fondo Editorial UCH.

Paro, V. H. (2002). Administraçao escolar: introduçao crítica. Sao Paulo: Cortez.

Paro, V. H. (Org.). (2006). A teoría do valor en Marx e a educaçao. Sao Paulo: Cortez.

Redes de "Círculos de Autoeducación Docente". (2013). Construyendo el Buen Vivir de los niños y niñas, desde la escuela. Lima: Instituto de Pedagogía Popular.

Vigotsky, L. S. (1979). El desarrollo de los procesos psíquicos superiores. Barcelona: Crítica.

Vigotsky, L. S. (1988). Pensamiento y lenguaje. México: Quinto Sol.

Wanderley, L.M. (Org.). (2005). A nova pedagogia da hegemonía. Estrategias do capital para educar o consenso. Sao Paulo: Xama. 